\title{
An Automatic Generation Method of Exploded View Based on Projection
}

\author{
Shuai Shao(0000-0002-8947-8849), Yufei Xing (0000-0001-8772-2038), Ligang Qu(0000-0002-3488-1331), Xin \\ $\mathrm{Li}(0000-0003-2896-3258)$ \\ School of Mechanical and Electrical Engineering, Shenyang Aerospace University, Shenyang, 110036. E-mail: \\ 2371915729@qq.com
}

Exploded view is a kind of schematic view which decomposes the assembly into parts and arranges according to certain rules to explain the product structural relationship and guide the relevant technical personnel to carry out product assembly or maintenance. It is necessary to consider the visibility constraints and explosion sequence rules to construct the exploded view. Manual creation will lead to repeated modification and low efficiency. In this paper, a projection based algorithm for automatic generation of exploded view is proposed. In this method, the explosion set of products is constructed based on disassembly matrix and the assembly sequence of complex products is represented as a set of ordered hierarchical exploded view, so as to ensure that the exploded view can clearly express the feasible assembly sequence. The projection interference detection method is proposed to ensure the visibility of the exploded view under a given angle of view, and the method of selecting the explosion direction is proposed to improve the compactness of the generated exploded view, and the explosion direction guideline is established to improve the readability of the exploded view. The algorithm has small storage space, fast search speed, and is not limited to a single specific perspective, so it is more general. The feasibility and efficiency of the algorithm are verified by an example of automatic generation of explosive drawing of fixture model.

Keywords: Exploded view, Projection, Oriented bounding box

\section{Introduction}

Exploded view is a kind of schematic view which decomposes the assembly into parts and arranges according to certain rules to explain the product structural relationship and guide the relevant technical personnel to carry out product assembly or maintenance.Compared with the two-dimensional assembly drawing, the exploded viewpresents the product structure in the form of three-dimensional axonometric projection, which can more intuitively show the assembly sequence of parts, and is easier to identify and operate with technicians.Different from the dynamic demonstration (video demonstration) of assembly process simulation, exploded view can be transmitted through traditional paper media or pictures in the form of two-dimensional stereo image, which greatly reduces the time and economic cost of assembly process expression.So it is widely used in various consumer products, electronic products, complex mechanical equipment and other fields[1].There are two ways to generate exploded view: man-machine interaction [2-3] and automatic generation. The former is generally applicable to products with less parts and general complexity. Some commercial CAD software also provides the related functions of manually creating product exploded view.In recent ten years, the research on automatic generation algorithm of exploded view has been put forward. In order to ensure that the exploded view can correctly express the assembly sequence, Li canlin et al. [4] used geometric interference rules to generate local exploded views in rectangular coordinates. Mohammad and Kroll [5] extracted the above graph from the product model to describe the assembly relationship of parts, and established conversion rules to realize the automatic generation of exploded views. Furthermore, the exploded distance and the perspective of the exploded view directly affect the visualization expression of the assembly sequence. Yu Jiapeng et al. [6] successively proposed an exploded distance algorithm based on axis aligned bounding box (AABB) and oriented bounding box (OBB); Zhao Hongfei et al. [7] adopted fixed direction hulls(FDH) makes the exploded distance of the parts more compact; $\mathrm{Li}$ et al. [8] proposed the exploded view with dynamic perspective, which enables the exploded view to switch the local exploded position with the movement of the view angle. In addition, considering that it is difficult to express all the details of complex product assembly by single exploded view.Agrawala et al. [9] proposed the design rules of step-by-step assembly guidance view; Vieilly et al. [10] used the bounding sphere to calculate the exploded distance of parts to generate hierarchical exploded view; Tatzgern et al. [11] expressed the corresponding assembly details through the enlarged local exploded 
view. Through the analysis of the above literature results, we can see that there are still two points to be improved in the problem of automatic generation of exploded view: first, only using bounding box to calculate the exploded distance can obtain a relatively compact exploded view, but due to the lack of consideration of the perspective of the exploded view.Therefore, the generated exploded view often appear the phenomenon that parts block each other in some given view angles (such as equiaxed or oblique axis side views), and this kind of "axonometric view" is usually the most concise and three-dimensional product assembly auxiliary drawing in engineering. In view of this kind of problem, new automatic generation method of explosive image is still to be proposed.On the other hand, the existing methods generally stipulate that the exploded direction is strictly consistent with the assembly direction, which greatly limits the flexibility of the layout of the exploded view; on the contrary, if the exploded direction is not constrained, the readability of the exploded view is not high, the assembly direction indication is not clear or even ambiguous.

Therefore, based on the existing literature research results, this paper proposes an algorithm for automatic generation of exploded view based on projection. This method aims at the automatic generation of exploded view under the specified view angle, and proposes an exploded direction selection and exploded distance calculation method based on projection interference detection.By solving the position coordinates of the parts in the exploded view, the exploded views without mutual occlusion between parts under the given view angle are automatically generated. MATLAB and Siemens NX system are used to verify the feasibility and efficiency of the algorithm.

\section{Description of exploded view generation rules}

\subsection{Exploded set partition rules}

Exploded view is an effective means to express the assembly relationship of products.However, it is difficult to express every assembly detail clearly when all parts are placed in a single exploded view for more complex products.Therefore, in engineering, the assembly process of a complex product is usually divided into several levels according to certain rules, and all parts required to be assembled in each layer are expressed by an exploded view, and finally the assembly process of the whole product is displayed by a set of orderly hierarchical exploded view. In order to construct hierarchical exploded view, this paper divides the assembly process of products $t_{i}$ by dividing all parts that can be assembled at the same time into one explosded set of products $s_{i}$, which divides the assembly process of products. Each explosded set generates an exploded view to represent a level of product assembly, which ensures that the exploded view correctly represents the geometric priority relationship of part assembly and effectively reduces the number of parts contained in a single exploded view so that the exploded view can accurately and clearly represent the feasible assembly sequence.

In this paper, the sampling Disassembly Matrix(DM) [12-13] is used to construct the explosion set of products.The disassembly matrix describes the interference relationship between each part in the assembly and other parts during its movement to infinity along the three orthogonal coordinate axis directions (hereinafter referred to as the orthogonal direction) in the absolute coordinate system of the assembly.The disassembly matrix is defined as follows:

Suppose assembly $\mathrm{P}$ is composed of $\mathrm{n}$ assembly unit body $P=\left\{p_{1}, p_{2}, \ldots, p_{N}\right\} \quad$.The interference between the part $p_{i}$ and the part $p_{j}$ in the process of moving along the $\mathrm{d}(d=+x,+y,+z)$ direction is expressed as $I_{d i j}(0 \leq i, j<n)$. If $i=j, I_{d i j}=0$;If $i \neq j$, the matrix is called the disassembly matrix $I M=\left[I_{d i j}\right]_{3 \times n \times n}$ of the assembly $\mathrm{P}$ in the orthogonal direction.

$$
I_{d i j}= \begin{cases}0 & p_{i} \text { moves in the d direction without collision interference with } p_{j} \\ 1 & p_{i} \text { moves in the } \mathrm{d} \text { direction with collision interference with } p_{j}\end{cases}
$$

According to the relativity of motion, the interference between $p_{j}$ and $p_{i}$ in $-d$ direction is the same as that between $p_{i}$ and $p_{j}$ when it is assembled along $d$ direction. The interference between the assembly of part $p_{i}$ along the $-d$ direction and the part $p_{j}$ is determined by $I_{d j i}$. Therefore, the interference matrix can be used to deduce the assembly interference relationship of the parts in $-d(-d=-x,-y,-z)$ three directions.

According to formula (1), the disassembly matrix $I M=\left[I_{d i j}\right]_{3 \times n \times n}$ is searched.If the part $p_{i}$ along the direction $d$ which does not interfere with other parts at the initial $t_{0}$ moment, the part can perform the exploded operation to construct the exploded set $s_{0}$ from all the parts that conform to the rule.After that, all the parts in the exploded set $s_{0}$ are disassembled and the matrix is searched again to get all the detachable parts at the next time $t_{1}$ as the exploded set $s_{1}$ .And so on until all parts are disassembled, the set $S=\left\{s_{0}, s_{1}, s_{2}, \cdots, s_{i}, \cdots, s_{m}\right\} \quad$ can be obtained.Each subset $s_{i}$ is the set of all parts that can be assembled at the same time at time $t_{i}$, that is, the exploded set $s_{i}$ at time $t_{i}$. If $S$ has $m$ subsets in total, then a total of $m$ exploded view need to be generated.In order to express the assembly process of products, the exploded view 
is generated for each subset of the set $S$ in reverse order. In addition to displaying the parts in the current exploded set, each exploded view also needs to display the parts that have been exploded to reflect the possible assembly relationship between parts. In this paper, the part with the largest volume in the exploded set is taken as the reference part (that is, the static part in the exploded view) so as to calculate the explode distance and determine the starting and ending coordinates of the exploded direction guideline.

\subsection{Engineering rules for exploded view generation}

The complexity of constraints and engineering rules is one of the difficulties in the automatic generation of exploded view.The following are some important rules for exploded view generation.

Rule 1: The exploded view should be able to reflect the geometric priority of product assembly;

Rule 2: The visibility of exploded view of complex products should be considered to show the assembly relationship of parts as clearly as possible;

Rule 3: Under the given angle of view, the parts in the exploded view have visibility, and there is no mutual occlusion;

Rule 4: The exploded direction of the parts should be able to reflect the assembly direction, and should be as simple as possible and easy to identify and operate with engineering and technical personnel;

Rule 5: The exploded view should be as compact as possible.

\section{Automatic layout algorithm of exploded view}

Aiming at the problem that parts shield from each other in view of given angle of view of exploded view, this paper comprehensively considers the position of parts in exploded view from three aspects: perspective of exploded view, exploded distance and exploded direction. The algorithm mainly includes projection interference detection, exploded distance calculation and exploded direction selection.

\subsection{Concept introduction}

According to the basic principle of computer graphics, it can be judged whether two objects are obscured from each other.After the object is projected to the window plane, it can be judged by whether the boundary boxes of the projection of objects intersect;At the same time, considering that the isometric side view (Hereinafter referred to as shaft side view ) is one of the most widely used views to present exploded view in static form.In this paper, the view of positive isometric side view is taken as the visual angel of exploded view.The exploded distance and exploded direction of the parts are calculated with the constraint condition that there is no mutual occlusion between the parts in the positive isometric side view, and then the exploded view is generated automatically.

Firstly, the boundary box of the part projected onto the isometric side view is constructed.In order to reduce the computational complexity of the projected bounding boxes and ensure that all the bounding boxes are convex polygons, take the direction of the part bounding box as the projection object (Hereinafter referred to as bounding box), As shown in Figure 1, let the bounding box of the part $p_{i}, p_{j}$ be respectively $B o x_{i}, B o x_{j}$, and the vertex set of the bounding box is respectively $V_{i}=\left\{V_{i 1}, V_{i 2}, V_{i 3}, V_{i 4}, V_{j 5}, V_{i 6}, V_{i 7}, V_{i 8}\right\}, V_{j}=\left\{V_{j 1}, V_{j 2}, V_{j 3}, V_{j 4}, V_{j 5}, V_{j 6}, V_{j 7}, V_{j 8}\right\}$. As shown in Figure 2, $\left(x_{i k}, y_{i k}, z_{i k}\right),\left(x_{j k}, y_{j k}, z_{j k}\right)$ is the coordinate value of two bounding box vertices $V_{i k}, V_{j k}$ respectively, Normalized with homogeneous coordinates as $\left(x_{i k}, y_{i k}, z_{i k}, 1\right),\left(x_{j k}, y_{j k}, z_{j k}, 1\right)$, then the coordinates of projection points $V_{i k}^{\prime}, V_{j k}^{\prime}$ in Axonometric view can be calculated according to formula (2):

$$
V_{1 k}^{\prime}=V_{1 k} \times T_{z}
$$

$T_{z}$ is a transformation matrix:

$$
T_{z}=\left[\begin{array}{cccc}
\cos \gamma & 0 & -\sin \gamma * \sin \alpha & 0 \\
-\sin \gamma & 0 & -\cos \gamma * \sin \alpha & 0 \\
0 & 0 & \cos \alpha & 0 \\
0 & 0 & 0 & 1
\end{array}\right]
$$

For the exploded view of isometric side view constructed in this paper,the coordinate axis of the part has the same angle with the axis side projection plane, and it is projected to the axial side projection plane. Therefore, take $\alpha=35^{\circ} 16^{\prime}, \gamma=45^{\circ}$ [14].

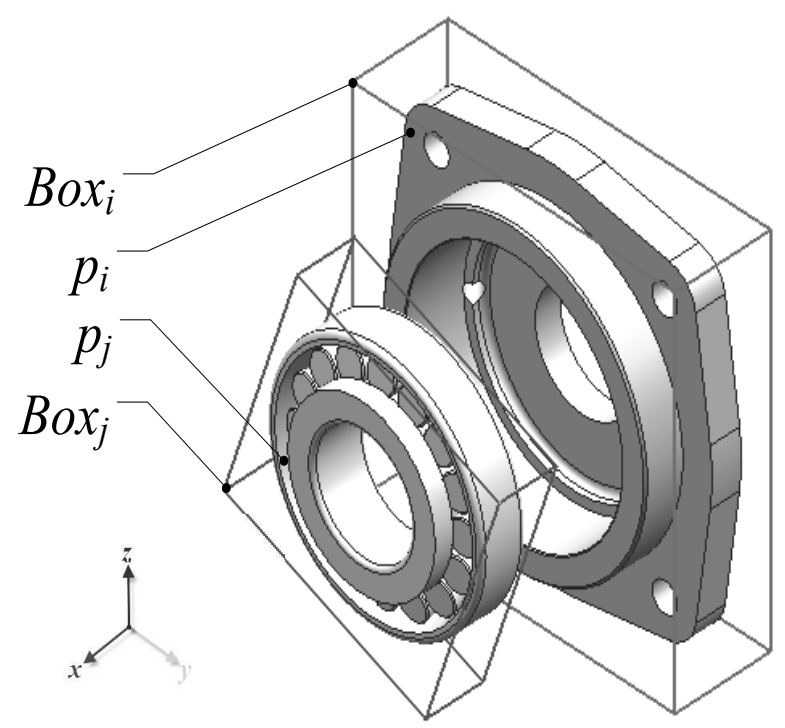

Fig.1 Parts and Bounding box in isometric projection

The set of projection points $p_{1}^{\prime}, p_{2}^{\prime}$ can be obtained by calculating the vertex set of each bounding box 
$V_{i}, V_{j}$ in Figure 2 according to formula (2). The polygon $p_{1}^{\prime}, p_{2}^{\prime}$ formed by the projection point is the bounding box formed by the part $p_{1}, p_{2}$ bounding box projected onto the axonometric view, as shown in $\mathrm{Fi}$ gure 3.

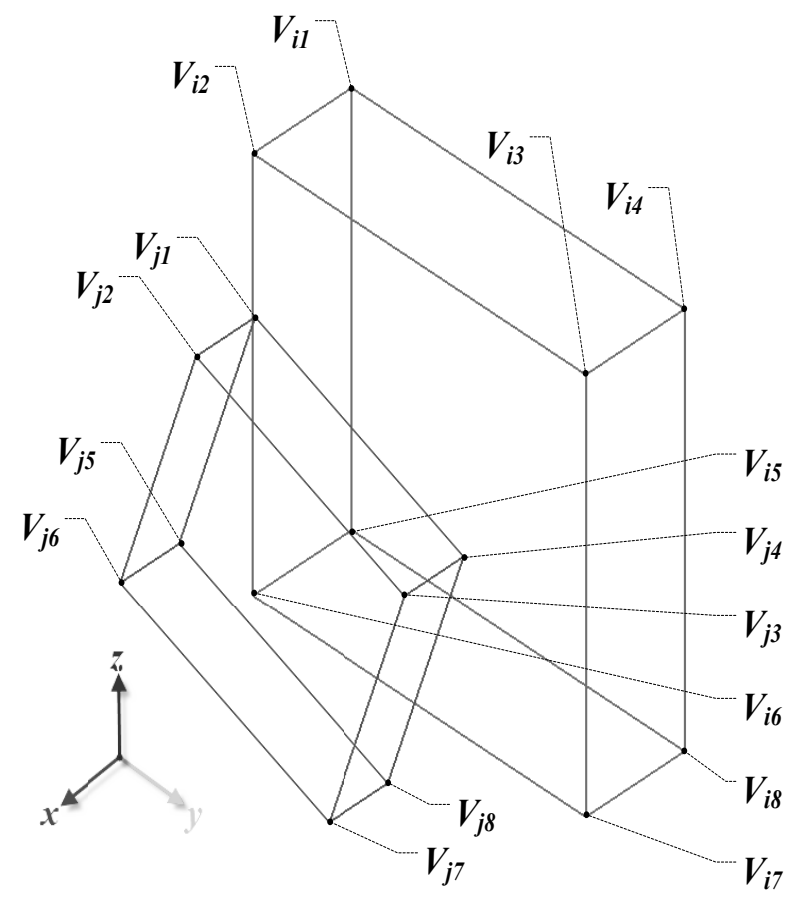

Fig. 2 vertexes of bounding box

\subsection{Projection interference detection}

It is inspired by the idea of judging the position relationship between point and polygon based on ray method in reference [15].In this paper, the boundary box intersection judgment method is constructed as follows: Take any vertex $V_{1 k}^{\prime}$ in the boundary box $p_{1}^{\prime}$ as the ray $k-k$,as shown in Figure 4,calculate the number of intersections of the rays $k-k$ with the boundary box ${ }_{2}^{\prime}$.According to the principle of topology, an even number of times a point intersects with a closed polygon indicates that the point is outside the polygo.On this basis, it can be determined that the point $V_{1 k}^{\prime}$ is outside the boundary box ${ }^{p_{2}}{ }^{\prime}$.By cyclic traversal, the number of intersections of all vertices in ${ }^{p^{\prime}}{ }^{1}$ with $p_{2}^{\prime}$ can determine whether the boundary box $p_{1}^{\prime}, p_{2}^{\prime}$ intersects or not .As shown in Figure 3, if there is an intersection area of the boundary box $p_{1}^{\prime}, p_{2}^{\prime}$, then there is projection interference in the boundary box $p_{1}^{\prime}, p_{2}^{\prime}$ obtained by projecting the boundary box(OBB) of the part ${ }_{1} p_{2} p_{2}$ on the axle-side view.Abbreviated as $p_{1}, p_{2}$ projection interference.Thus, it can be judged that the parts are obscured in the view angle of axle-side view.

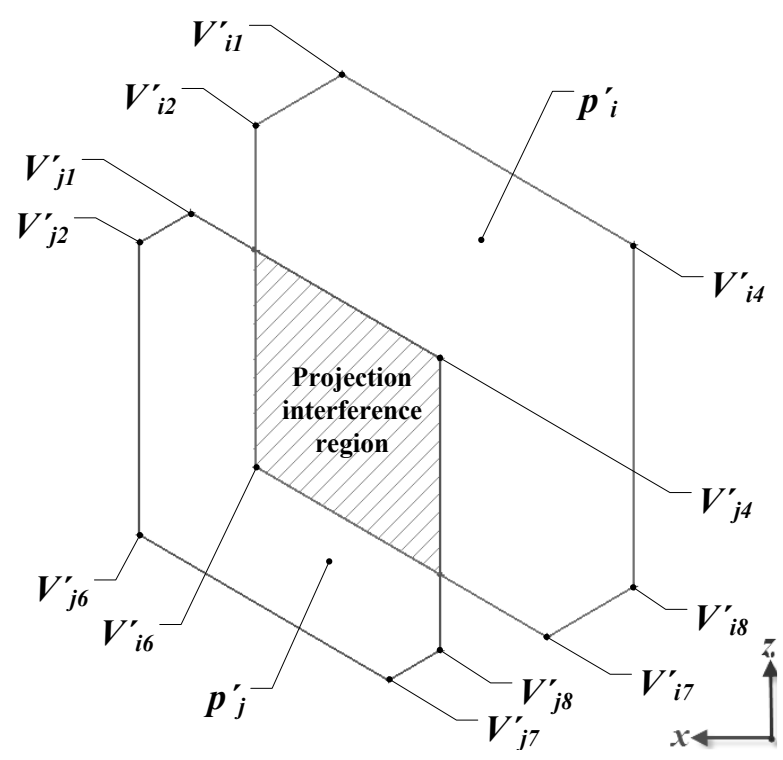

Fig.3 bounding box

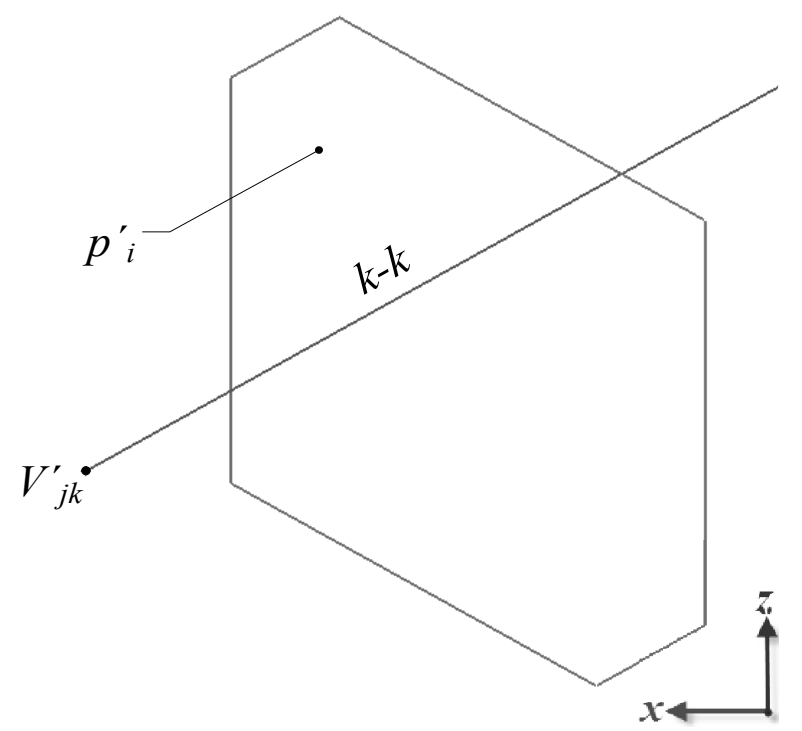

Fig.4 ray method

\subsection{Calculation of exploded distance}

Since the shielding of the part from a given exploded angle of view is considered, the previous method of calculating the exploded distance only by the size of the part enclosure box needs to be improved. The calculation method for explosion distance of parts in this paper is as follows:Firstly,the part ${ }^{p_{j}}$ is along the exploded direction in stepping-mode.Every stepping can update the bounding box coordinates of the part ${ }^{p_{j}}$ and calculates the new projected bounding box.Then, according to the method given in Section 2.2 , judge the intersection of boundary boxes of parts $p_{i}, p_{j}$ to further judge whether there is projection interference in parts ${ }^{p_{i}, p_{j}}$.If projection interference still exists, i.e. ${ }^{p_{j}}$ still obscures the part ${ }^{p_{i}},{ }^{p_{j}}$ continues to step in the exploded direction. Otherwise, stop and 
use the accumulated distance of step movement as the exploded distance of the part $p_{i}$.For the parts given in Figure 1, the final explosion results are shown in Figure 5.(Take $p_{i}$ as the reference part).

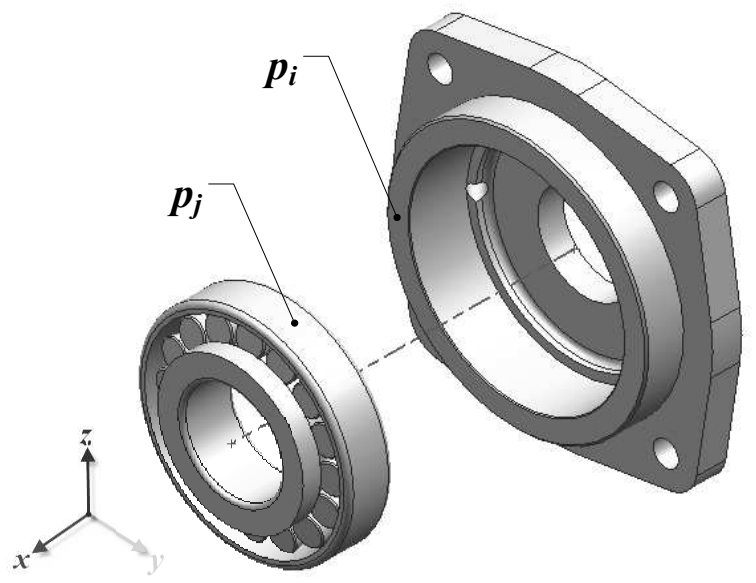

Fig.5 Expolded view of fig. 1

\subsection{Expansion in exploded direction}

The exploded direction of the existing exploded view strictly follows the disassembly direction of the parts. In fact, appropriate expansion of the exploded direction of parts can obtain more compact and reasonable layout effect. Therefore, this paper extends the exploded direction to the orthogonal direction, which not only ensures the indication of the assembly direction, but also improves the rationality of the layout. In this paper, rules for selection of exploded direction are designed:

Firstly, taking the direction of disassembly as the initial selection of the explosion direction of the part, in which the part moves with the preset exploded step length.If projection interference still exists with all parts that have completed the explosion after the part step times have reached the preset upper limit, the part re-selects the direction of explosion and continues to step .The principle of screening new explosion direction is to get away from projection interference area as soon as possible. However, since the transformation matrix $T_{z}$ between the vertex of the part enclosure box and the vertex of the projection boundary box is singular, it is difficult to reverse the vertex of the boundary box through the vertex coordinate of the boundary box.Therefore, this paper indirectly determines the new direction of explosion by comparing the projection position of the part boundary box to $x y, y z, z x$ three planes.Specific as follows: Set part $p_{q}$ as the finished exploded part. $p_{r}$ still has projection interference with $p_{q}$ after reaching the upper limit of the number of steps in the direction of disassembly.The vertices of bounding box of parts $p_{q}$ and $p_{r}$ are projected onto the plane $z x$ respectively, and the set of projection points $\left\{V_{q 1}^{n z}, V_{q 2}^{n}, V_{q 3}^{n,}, V_{q 4}^{n z}\right\}$, $\left\{V_{r 1}^{n z}, V_{r 2}^{n z}, V_{r 3}^{n}, V_{r 4}^{n z}\right\}$ forms the boundary box $p_{q}^{n z} p_{r}^{n=2}$ respectively.As shown in Figure 6,by ordering the coordinate values, we can see that $p_{q}^{\alpha z z} \cap p_{r}^{\mathrm{kzz}} \neq \varnothing$ the boundary boxes have an intersection area. Calculate separately the distance $p_{r}^{\text {nzz }}$ needs to move away from part $p_{q}^{\text {ng }}$ in $\{ \pm x, \pm z\}$ direction.Sort by the shortest moving distance,we can get $\left|p_{r}^{\min \_x}-p_{q}^{\max \_x}\right|<\left|p_{r}^{\max z}=-p_{q}^{\min \_}=\right|<\left|p_{r}^{\min \_z}-p_{q}^{\max =}=\right|<\left|p_{r}^{\max -x}-p_{q}^{\min \_x}\right|$ Further, by this method, the projection position relation of the vertex of the bounding box in the $y z, x y$ plane can be similarly compared, and it is finally determined that the part $p_{q}$ preferentially selects $+x$ as the new exploded direction. As shown in Fig. 7 (a),set parts as basic parts.Parts $\left\{p_{1}, p_{2}, p_{3}, p_{4}\right\}$ explode in sequence as exploded sets.Initial exploded direction is disassembly direction $+z$.Suppose that the upper limit of the initial number of stepping exploding along the disassembly direction is the ratio of the length of the part bounding box in that direction to the exploded step length.It is obvious that the size of part $p_{5}$ in $+z$ direction is large, and the projective interference exists between parts $p_{1}, p_{2}, p_{3}, p_{4}$ and $p_{5}$ after the exploded movement of part $p_{1}, p_{2}, p_{3}, p_{4}$ along $+z$ direction reaches the upper limit of stepping times.Thereafter $p_{1}, p_{2}, p_{3}, p_{4}$ reselects the direction of explosion according to the method given in this section, and the expansion results of exploded direction are shown in Fig. 7(b).After the exploded direction of all parts is re selected, not only can the mutual shielding be avoided, but also the more compact exploded view can be obtained.

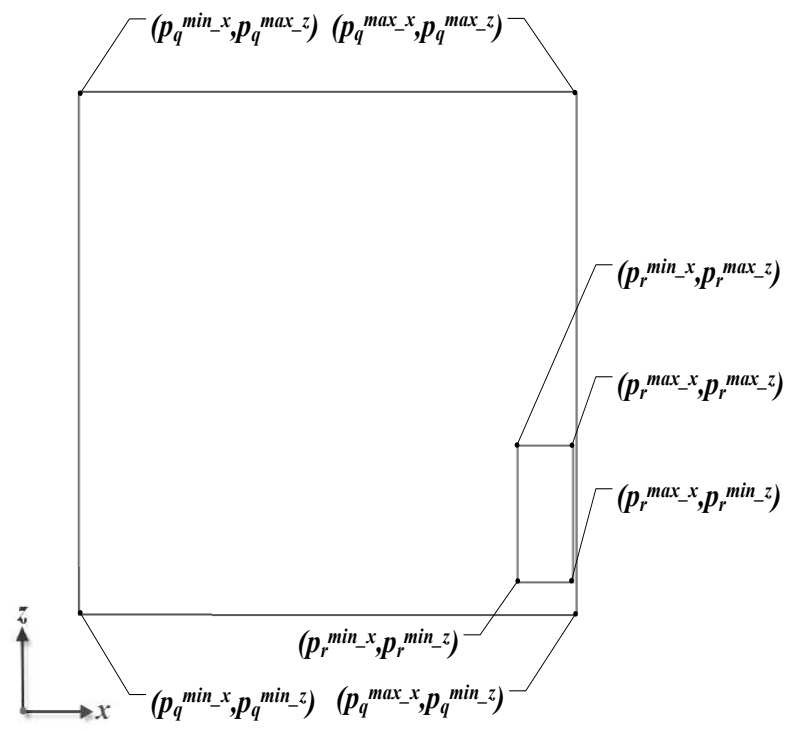

Fig.6 Expolded direction select based on bounding box 


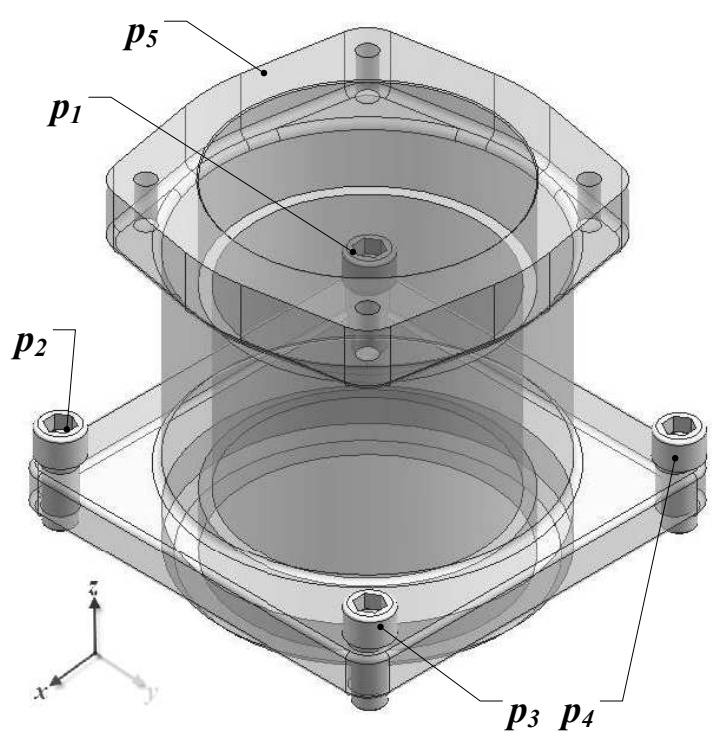

(a) Initial state

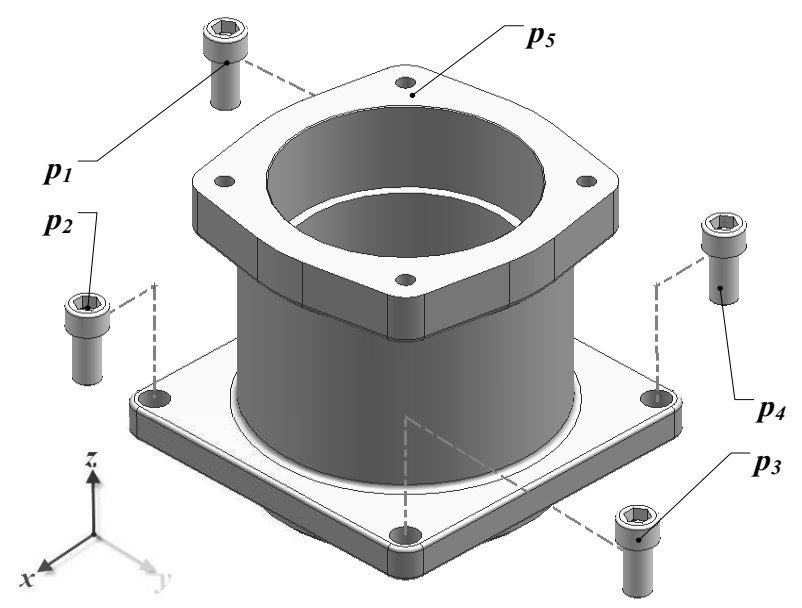

(b) exploded direction selected by the proposed method

Fig. 7 Expolded view compare with different exploded direction select method

In addition, to ensure that the assembly position of parts can be clearly expressed in the exploded view.The algorithm stipulates that exploded direction selected by a part can not be the same as the opposite direction of all previously selected exploded directions of the part and is assisted by exploded direction guideline. The guideline is a line segment connecting the exploded position of the part with the assembly position, which is used to represent the path of the part moving from the position in the exploded view to the assembly position. The starting point and end point of the guildline are the center points of the bounding box of the part in the exploded position and the assembly position respectively. Since the selection of explosion direction is limited to the orthogonal direction only, setting the direction of the guideline is also limited to the orthogonal direction. As shown in the dotted line in Figure 8.

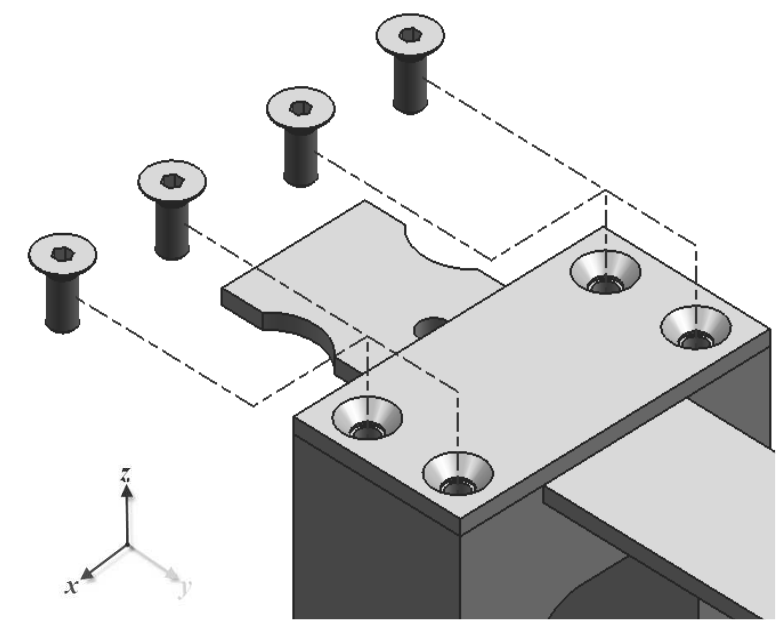

Fig. 8 Exploded view guideline generation by the method proposed in this paper

\subsection{Algorithm flow}

The flow chart of the algorithm for automatic generation of exploded view presented in this paper is as follows:

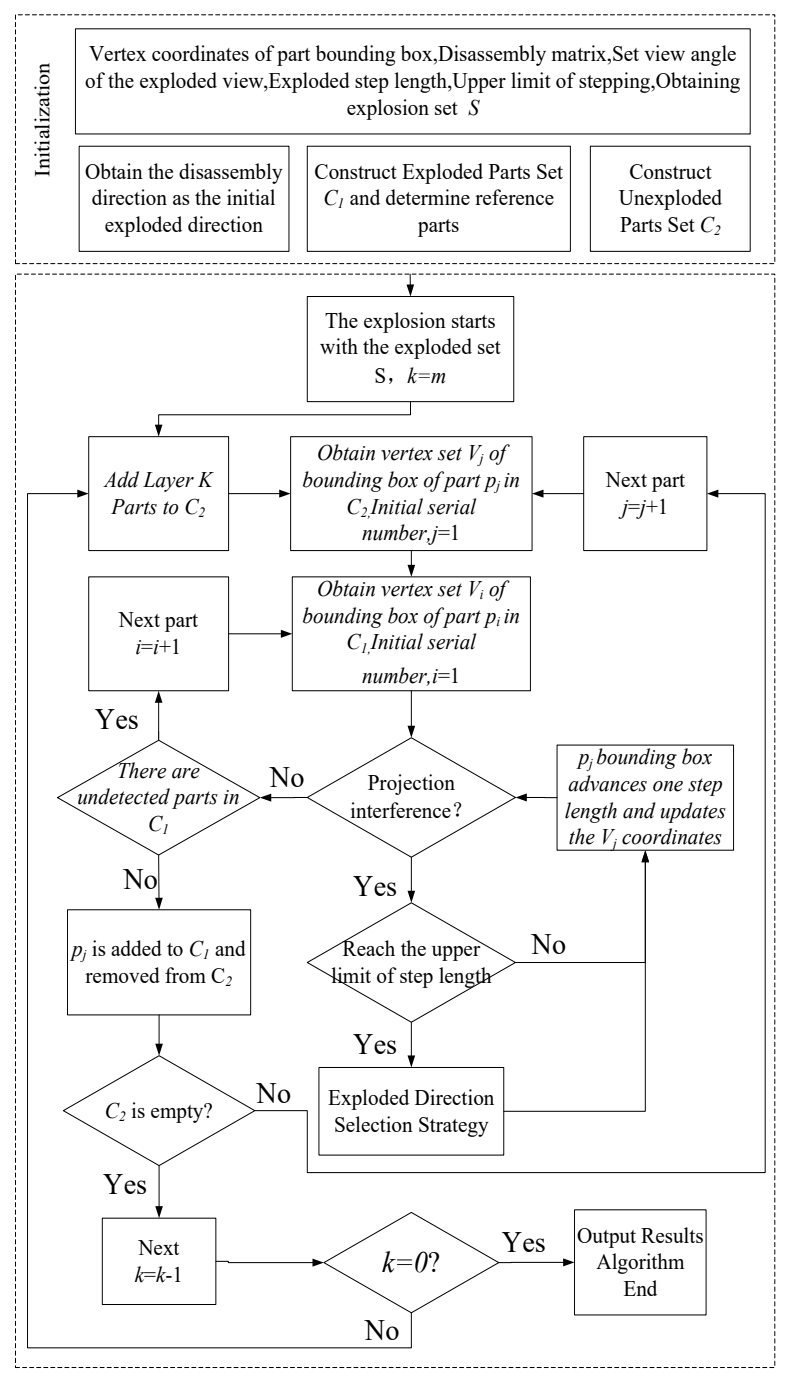

Fig.9 The flow diagram of algorithm 


\section{An example and analysis of automatic ge- neration of exploded view}

The test in this paper is based on MATLAB and UG / open API function joint implementation, both through text files to achieve data exchange.As shown in Figure 10.Firstly, the disassembly matrix of assembly model and the bounding box information of parts are extracted from Siemens NX 3D design software.Then combined with the preset exploded angle, exploded step length and the upper limit of stepping times as the input data into Matlab, the product explosion set is divided by the automatic explosion generation algorithm based on projection proposed in this paper.The exploded distance and direction of parts in each set are calculated in turn, and the coordinates of each part guideline are determined.Then, the calculation results are output to Siemens NX for three-dimensional exploded view display, so as to realize the visualization of exploded view.

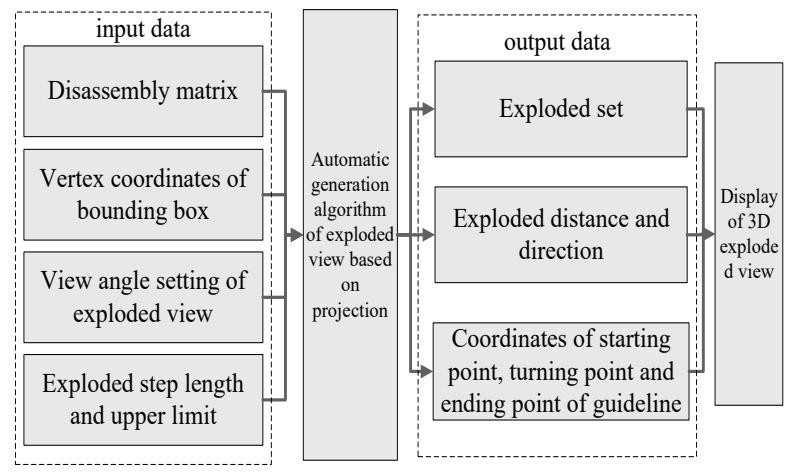

Fig.10 The diagram of exploded view automatic generation

In this example, an assembly fixture model is shown in FIG. 11 (e) (41 parts in total) to verify the effectiveness of the algorithm proposed in this paper.The model is divided into four explosion sets by the extracted disassembly matrix, that is to say, the whole assembly process is displayed by four exploded view, and each exploded view is generated from the perspective of positive equiaxed side view.Set the moving step of each explosion to $5 \mathrm{~mm}$. The upper limit of the exploded stepping times is the ratio of the length of the bounding box of the part along the disassembly direction to the step length.Each part first explodes along the disassembly direction. Then, if there is still projection interference with other parts in the exploded view, the exploded direction is expanded according to the method given in Section 2.4.The exploded view generated by all explosion sets are shown in Fig. 11 (a) (b) (c) (d).All parts in exploded view are not covered by each other and have good visibility. At the same time, because the exploded direction of the parts is not limited to the disassembly direction, the compactness of the exploded view is effectively improved.The results of the exploded view can meet the requirements of the exploded view generation rules mentioned in section 1.2 , which verifies the feasibility of the algorithm.The algorithm runs on the Dell (t1700) workstation (CPU Quad Core i7, $2.8 \mathrm{GHz}$, memory 8 GB). In Siemens NX 10.5, UG / open API function is used to develop the exploded movement and guideline drawing function of parts, and the output results are displayed.Because all operations are carried out in MATLAB, the operation time of generating all exploded views is only $85 \mathrm{~s}$, which has high efficiency.

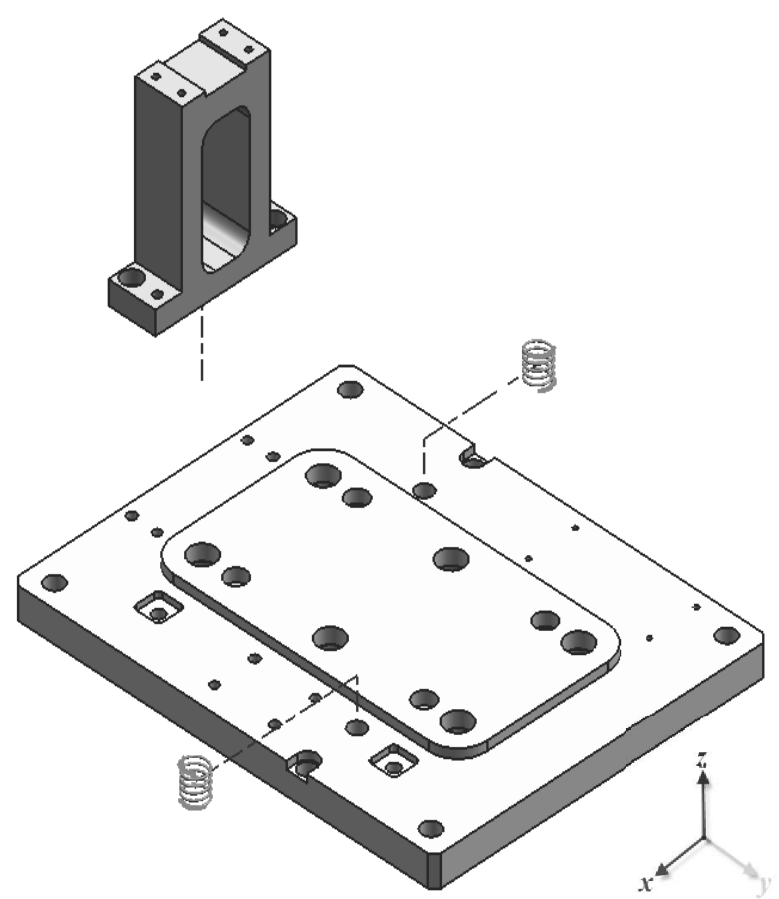

(a) first level

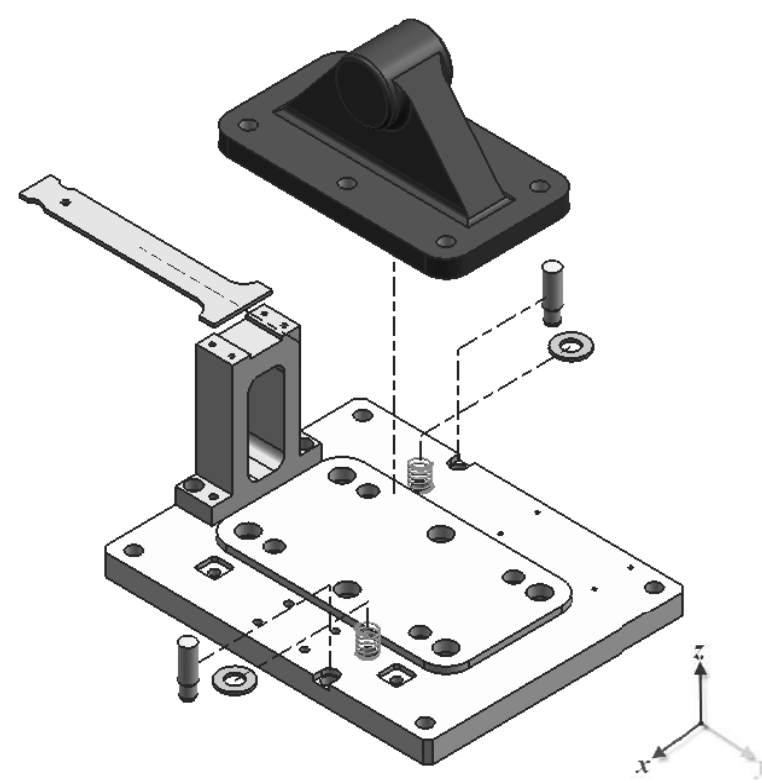

(b) second level 


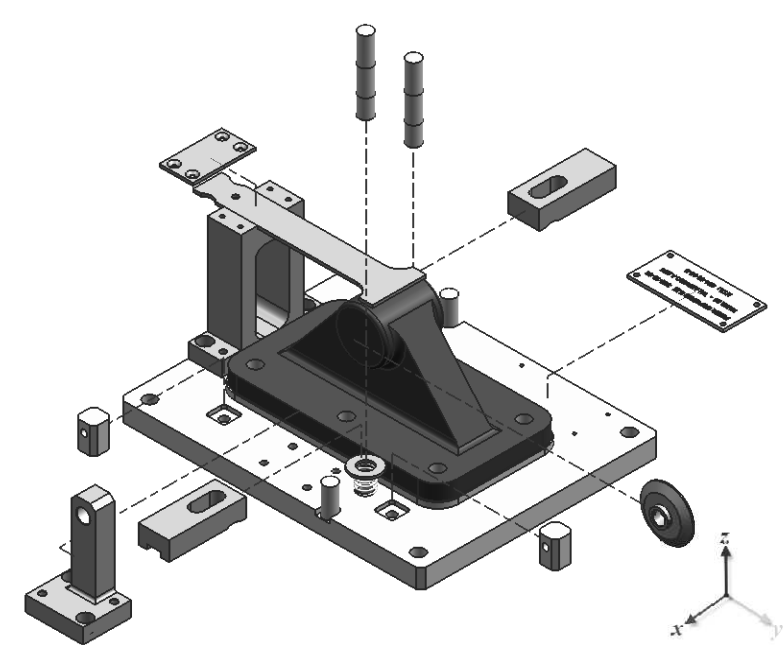

(c) third level

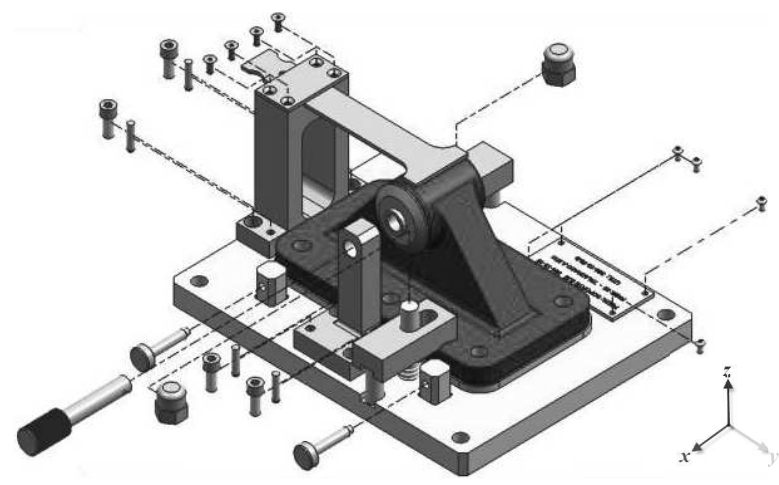

(d) forth level

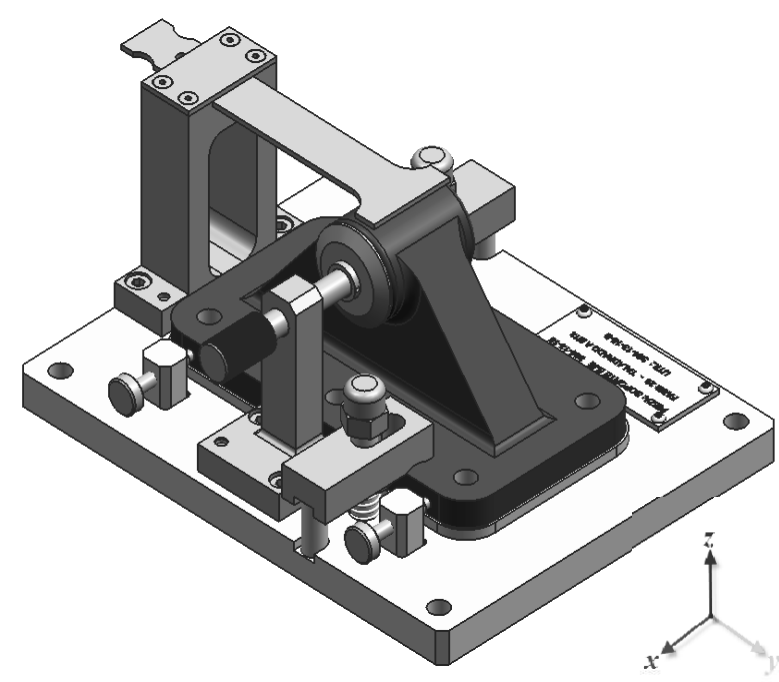

(e) Assembly model of a fixture

Fig.11 The exploded view automatic generation by the algorithm proposed in this paper

\section{Conclusion}

An automatic generation algorithm of exploded view based on projection is proposed, which can avoid covering each other and ensure the visibility of exploded view under given angle of view. The algorithm proposed in this paper is not limited to the exploded view with equal axis side angle, but also can be effectively applied to the automatic generation of exploded view with any given angle of view.So it is more general.

A method for constructing product exploded set based on disassembly matrix is proposed.It is more engineering meaningful to show the assembly process of complex products by automatically generating orderly hierarchical exploded view. The application of bounding box reduced the computational complexity, so that the algorithm still has high computational efficiency while guaranteeing the visibility of exploded view.

A new method for selecting exploded direction expansion is proposed to make exploded view more compact. At the same time, the selection of exploded direction is limited to the orthogonal direction, and the exploded direction guideline is used for auxiliary explanation, so that the exploded view can still clearly express the assembly relationship.

\section{References}

[1] COOK H T, FETTERMAN K D, WHITE D E. (2013).Interactive exploded view diagram ordering tool. US.

[2] MINAMI S, ISHIDA T, SHINOTSUKA Y, et al.(1997) .Apparatus for producing exploded view and animation of assembling and method thereof. US.

[3] KAWAI M. (2003).Exploded view automatic creation apparatus, storage media and methods.

[4] CANLIN LI, MING CAI, RUOFENG TONG, et al.(2004). Assembly sequence planning based on rule and exploded view . Journal of computer aided design and graphics, 16(8): 11061113.

[5] MOHAMMAD R, KROLL E. (1993).Automatic generation of exploded view by graph transformation. Artificial Intelligence for Applications. Proceedings., Ninth Conference on, 1993: 368374.

[6] JIAPENG YU,JIANXI WANG. (2016).Automatic generation method of hierarchical exploded view based on recursive loop. Journal of Mechanical Engineering, 52(13): 175-188.

[7] HONGFEI ZHAO, QI ZHANG, HAITAO WANG, et al. (2015). Automatic generation method of part exploded view based on synchronous constraint release . China Mechanical Engineering, 26(1): 70-73.

[8] LI W, AGRAWALA M, CURLESS B, et al.(2008). Automated generation of interactive 3D exploded view diagrams. Acm Transactions on Graphics, 27(3): 1-7. 
[9] VIEILLY L, DUMOULIN C, PATRIARCA C, et al. (2013).Computer system and method for providing exploded views of an assembly. US.

[10] AGRAWALA M, PHAN D, HEISER J, et al.(2003). Designing effective step-by-step assembly instructions. Acm Transactions on Graphics, 22(3): 828-837.

[11] TATZGERN M, KALKOFEN D, SCHMALSTIEG D. (2011).Multi-perspective compact explosion diagrams. Computers \& Graphics, 35(1): 135-147.

[12] YUFEI XING, CHENGEN WANG, JIAPENG YU. (2012).Automatic generation of exploded view based on ant colony algorithm .
Journal of Northeast University (NATURAL SCIENCE EDITION), 33(6): 883-886.

[13] YUANMAOHUANG, CHUNTINGHUANG. (2002).Disassembly matrix for disassembly processes of products. International Journal of Production Research, 40(2): 255-273.

[14] FEI TANG, WEI YANG, CHANGLI CHA. (2016).MATLAB Realization of Three-dimensional Projection Transform . Journal of Anbui University of science and technology, 36(2): 36-39.

[15] ZEGEN WANG. (1999).Improvement of the relationship between the determination point and polygon inclusion by ray method. Journal of Surveying and Mapping Science and technology, (2): 54-56. 